\title{
Nucleons near the QCD deconfinement transition
}

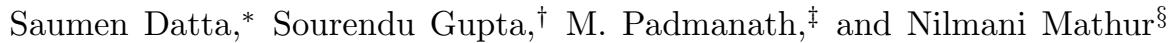 \\ Department of Theoretical Physics, \\ Tata Institute of Fundamental Research, \\ Homi Bhabha Road, Mumbai 400005, India. \\ Jyotirmoy Mait] \\ Department of Physics, Barasat Government College, Barasat, Kolkata 700124, India.
}

\begin{abstract}
Using non-perturbative lattice method we studied hadronic screening correlators above and immediately below the deconfinement transition temperature, $T_{c}$, in the quenched approximation with lattice spacing of $1 /(8 T)$ using clover improved Wilson fermions. Simulations were performed at temperatures $T / T_{c}=0,0.95$ and 1.5. Mesonic screening correlators show no statistically significant thermal effects below $T_{c}$, and clear evidence for weakly interacting quarks above $T_{c}$. Baryon screening correlators yield similar physics above $T_{c}$, but show precursor effects for chiral symmetry restoration below $T_{c}$.
\end{abstract}

PACS numbers: 12.38.Mh, 11.15.Ha, 12.38.Gc

\section{INTRODUCTION}

Experiments with relativistic heavy-ion collisions are beginning to probe the finer details of non-perturbative predictions of QCD [1]. This is possible because fireballs produced in these collisions come close to thermal equilibrium [2], and lattice QCD techniques can be used to predict expectation values of observables under these conditions [3]. The question of why the fireballs come into equilibrium, i.e., why relaxation times are small [4], remains outside the direct purview of lattice computations for now. Even so, one can ask why thermodynamics is applicable [5]. The answer can be found by testing whether any static correlation length in a QCD medium, $\xi$, (which is the inverse of a screening mass, $\mu$ ) is much smaller than the size of the fireball.

The only correlations which persist in the equilibrium thermodynamic system are static spatial correlations. These correlation functions are measured by introducing static probes into the equilibrium plasma and measuring the response of the medium. This response depends on the quantum numbers carried by the probes; so one can classify static correlators as glueball-like, meson-like and baryon-like probes with the usual quantum numbers of these quantities corrected for the fact that the static spatial symmetries are different from the Poincare group. The group theory of these correlators was worked out for glueballs [6 [8] and mesons [9]. In Appendix $\mathrm{A}$ we extend the representation theory to spin- $1 / 2$ baryons.

Meson-like screening masses have been studied in QCD in great detail 10 17. While we continue these studies

\begin{tabular}{|c||c|c||c|c|}
\hline$\frac{m_{\pi}}{T_{c}}$ & $\frac{\mu_{V}}{m_{V}}$ & $\frac{\mu_{N_{+}}}{m_{N_{+}}}$ & $\frac{\mu_{A V}-\mu_{V}}{m_{A V}-m_{V}}$ & $\frac{\mu_{N_{-}}-\mu_{N_{+}}}{m_{N_{-}}-m_{N_{+}}}$ \\
\hline 2.20 & $0.97 \pm 0.02$ & $1.05 \pm 0.05$ & $0.86 \pm 0.16$ & $0.75 \pm 0.10$ \\
1.99 & $0.94 \pm 0.03$ & $1.07 \pm 0.04$ & $0.89 \pm 0.22$ & $0.68 \pm 0.12$ \\
\hline
\end{tabular}

TABLE I: Thermal shifts in masses and mass splittings at $T=0.95 T_{c} ; \mu_{H}$ denotes the screening length in the hadronic channel $H$, and $m_{H}$ the mass at $T=0$. We find that $\mu_{H}=m_{H}$ within $95 \%$ confidence limits, indicating no significant thermal effects in the vector meson, $\mathrm{V}$ and nucleon, $N_{+}$. Nor do we observe a thermal shift in the mass splittings between the $\mathrm{V}$ and the AV (axial vector) mesons. However, the splitting between the $N_{+}$and its parity partner, $N_{-}$, changes at finite temperature, and is a precursor to the restoration of chiral symmetry before the QCD phase transition.

\footnotetext{
*Electronic address: saumen@theory.tifr.res.in

${ }^{\dagger}$ Electronic address: sgupta@theory.tifr.res.in

¥Electronic address: padmanath@theory.tifr.res.in

§Electronic address: nilmani@theory.tifr.res.in

๑Electronic address: jyotirmoy.maiti@gmail.com
} 
here, the main focus of this work is the baryon sector. Baryon-like screening correlators were studied in the hightemperature phase [10, 11], but there have been no follow-up studies in recent years, nor have these been extended to the low-temperature phase till now. Since these provide important inputs to the study of baryon-number fluctuations, which is of much interest in the experimental search for the critical point of QCD, we reboot the study of these correlators.

Above $T_{c}$ we verify that baryonic screening lengths support the evidence for weakly interacting quarks which has been gathered over the years with meson screening correlators. Below, but very close to, $T_{c}$ we find no evidence of any finite temperature effects on the masses of the lowest mesonic resonances. However, we find that in the nucleon sector the mass of the opposite parity nucleon moves closer to the ground state (see Table I). These observations not only constrain models of quantum hadrodynamics [18], but could also have implications for the analysis of heavy-ion collision data.

The paper is organized as follows. In Section II we discuss technical details related to the simulation, inversion and analysis, including the details of interpolating operators used and the asymptotic fit forms. In Section III and Section [V] we display the results in detail. Section $\nabla$ summarizes and concludes the work done in this paper. Various technical details are presented in appendices.

\section{RUNS AND MEASUREMENTS}

\begin{tabular}{|c|c|c|c|c|c|}
\hline$T / T_{c}$ & $N_{\tau} \times N_{s}^{3}$ & $\beta$ & $N_{\text {conf }}$ & $c_{S W}$ & $\kappa$ \\
\hline 0 & $32 \times 16^{3}$ & 6.03 & 71 & 1.7333 & $0.1345,0.1347$ \\
0.95 & $8 \times 32^{3}$ & 6.03 & 94 & 1.7333 & $0.1345,0.1347$ \\
1.5 & $8 \times 32^{3}$ & 6.332 & 67 & 1.5667 & $0.1345,0.1350,0.1355$ \\
\hline
\end{tabular}

TABLE II: The simulation and measurement parameters used in this work. $\beta$ is the bare coupling for the Wilson gauge action, $c_{S W}$ is the clover coefficient (determined non-perturbatively in [19]), and $\kappa$ is the hopping parameter in the improved Wilson-Dirac operator.

We measured correlation functions of operators with meson and baryon quantum numbers built out of cloverimproved Wilson quarks [20] in zero and finite temperature ensembles of configurations generated using the Wilson gauge action. The finite temperature configurations were generated on lattices with lattice spacing $a=1 /(8 T)$ with $T=0.95 T_{c}$ and $1.5 T_{c}$. The spatial size of the lattice, $L$, was tuned such that $L T=4$. We use the notation $V=L^{3}$, $V_{4}=V / T, N_{t}=1 /(a T)$ and $N_{s}=L / a$. Zero temperature measurements were carried out on $(2 L) \times L^{3}$ lattices with coupling $\beta=6.03$, which corresponds to the same lattice spacing as the finite temperature run below $T_{c}$ [21, 22]. We will describe later that this meant that $L$ was 8.4 times the Compton wavelength of the lightest state on the lattice, as a result of which finite volume effects are under control. A zero temperature study at $\beta=6.332$, corresponding to the thermal ensemble above $T_{c}$ was not made, but the corresponding choice of $\kappa$ was made so that we could use a previous study [23]. Details of the simulation and measurement parameters are tabulated in Table II]. With the choices of $\kappa$ listed for $\beta=6.332$, the values of $m_{\pi} / T_{c}$ are $1.52,2.58$, and 3.31 respectively. For the other parameters, the results of the mass measurements are described later.

The gauge configurations were generated by a combination of one heat bath and three overrelaxation updates per Monte Carlo (MC) step. The first 2000 MC steps were discarded to ensure equilibration of the lattices. Subsequently, measurements were performed once in $200 \mathrm{MC}$ steps, which is approximately 30 to 100 times the autocorrelation time of the action. As a result, it took $4 \mathrm{CPU}$ hours to generate a single configuration on our largest lattice.

The measurement of hadronic correlation function requires the inversion of the Dirac operator. This was done by a conjugate gradient algorithm which stopped when the residual dropped below $\left(V_{4} / a^{4}\right) \times 10^{-16}$. Typically this was found to take about 2500 conjugate gradient steps, and about 6 hours of CPU time, for the smallest pseudo scalar mass used in our calculations below $T_{c}$. The computations above $T_{c}$ are substantially cheaper; the inversions converged in approximately $0.67 \mathrm{CPU}$ hour for our lightest pseudo scalar mass.

The zero momentum correlation functions are

$$
S_{H}(t)=\sum_{\mathbf{x}}\left\langle H^{\dagger}(\mathbf{x}, t) H(\mathbf{0}, 0)\right\rangle, \quad \text { where } \quad H(\mathbf{x}, t)=\bar{\psi}(\mathbf{x}, t) \Gamma_{H} \psi(\mathbf{x}, t),
$$

where $\psi(\mathbf{x}, t)$ is the quark field at time $t$ and spatial point $\mathbf{x}$ (we will also use the component notation $\mathbf{x}_{1}=x, \mathbf{x}_{2}=y$ and $\mathbf{x}_{3}=z$ ). The sum over all spatial sites of the point-to-point correlator projects on to zero spatial momentum. All Dirac, flavour, and colour indices are summed. $\Gamma_{H}$ is an appropriate Dirac, flavour matrix which gives the quantum 
numbers of the meson $H$. In actual practice, we replaced the point source of eq. (1) by a wall source, in order to control the statistics better. This required gauge fixing; we fixed to the Coulomb gauge. The effect on the extraction of screening masses is discussed in Appendix B

We used $\Gamma_{P S}=\gamma_{5}$ for the isovector pseudoscalar (PS), $\Gamma_{S}=1$ for the isovector scalar $(\mathrm{S}), \Gamma_{V}=\gamma_{i}$ for the vector $(\mathrm{V})$ and $\Gamma_{A V}=\gamma_{i} \gamma_{5}$ for the isovector axial vector (AV) channels. For the $T=0$ measurements, we considered propagation in the time direction as shown in eq. (11), and since all the orthogonal directions are equivalent, we summed the $\mathrm{V}$ and AV propagators over the three polarizations $i=1,2,3$. For the screening correlators all the polarizations of the $\mathrm{V}$ and $\mathrm{AV}$ are not equal, and we summed over only $i=1$ and 2 .

The lattice mesonic currents need to be renormalized to connect them with the continuum currents. For quarks of mass $m_{q}$, the lattice operators, $H(\mathbf{x}, t)$, are multiplicatively renormalized to

$$
Z_{H}\left(g^{2}\right)\left(1+b_{H}\left(g^{2}\right) a m_{q}\right) H(\mathbf{x}, t)
$$

where $a$ is the lattice spacing, $g^{2}=6 / \beta$, and $a m_{q}=\left(\kappa-\kappa_{c}\right) / 2$. We use the results in the $\overline{M S}$ scheme where the renormalization constants are determined at the scale of $1 / a$. For non-perturbatively improved clover fermions, the factors $Z_{V, A V}$ and $b_{V}$ have been calculated non-perturbatively [24], where the following interpolating formulae are found:

$$
Z_{V}=\frac{1-0.7663 g^{2}+0.0488 g^{4}}{1-0.6369 g^{2}}, \quad Z_{A V}=\frac{1-0.8496 g^{2}+0.0610 g^{4}}{1-0.7332 g^{2}}, \quad b_{V}=\frac{1-0.6518 g^{2}+0.1226 g^{4}}{1-0.8467 g^{2}} .
$$

For the other coefficients, we use the expression from the one-loop, tadpole improved perturbation theory [25]:

$$
Z_{P S, S}=u_{0}\left(1-z_{P S, S} \tilde{g}^{2}\right)
$$

where $u_{0}$ is the tadpole factor, $\langle P\rangle=u_{0}^{4}, \tilde{g}^{2}=g^{2} / u_{0}^{4}$, and the one-loop coefficients $z_{P S, S}$ as obtained from [26] are $z_{P S}=0.107-0.019 \tilde{c}_{S W}+0.017 \tilde{c}_{S W}^{2}$ and $z_{S}=0.026+0.065 \tilde{c}_{S W}-0.012 \tilde{c}_{S W}^{2}$ where $\tilde{c}_{S W}=u_{0}^{3} c_{S W}$. The one-loop order tadpole-improved forms of $b_{H}$ can be written as

$$
b_{H}=\frac{1}{u_{0}}\left(1+b_{H}^{1} \tilde{g}^{2}\right) .
$$

where one obtains from the calculations of [27] the values $b_{P S}^{1}=0.109, b_{S}^{1}=0.070$, and $b_{A V}^{1}=0.069$, computed for the tree-level $\tilde{c}_{S W}=1$.

Masses and screening masses can be obtained without renormalization. They were estimated, as usual, by fitting to an assumed cosine-hyperbolic form, and looking for agreement with effective masses. These imply assumptions about the spectral function at zero and finite temperatures, and we discuss these in more detail in Appendix B Statistical errors on masses are obtained by a jackknife procedure. The propagation of statistical errors is done by jackknife when they could be correlated, and by adding in quadrature when they are independent. The scalar propagator has strong quenching artifacts. This prevents the use of these correlators in estimating masses in the usual way (see Appendix C).

Chiral symmetry restoration in the high temperature phase of QCD is signalled by pairwise equality of correlators which are related to each other by parity. The observation of some breaking of this symmetry has been an issue of some interest recently [16]. Here we introduce a new measure for this symmetry-

$$
R_{H}=\frac{1}{N_{t}-1} \sum_{t=1}^{N_{t}-1} \frac{\left\langle S_{H_{+}}(t)-S_{H_{-}}(t)\right\rangle}{\left\langle S_{H_{+}}(t)+S_{H_{-}}(t)\right\rangle},
$$

where $S_{H_{ \pm}}(t)$ are parity partner correlators (for the screening correlators $t$ is replaced by $z$ and $N_{t}$ by $N_{s}$ ), and the angular brackets are averages over the gauge ensemble. The correlators at $t=0$ are left out of the sum to avoid problems with time doublers. We will use the convention of taking the positive parity partner for the label $\mathrm{H}$. When chiral symmetry is broken we expect $R_{H} \simeq \mathcal{O}(1)$.

For the nucleon operator we used

$$
N_{\alpha}(\mathbf{x}, t)=\varepsilon_{a b c}\left(C \gamma_{5}\right)_{\beta \delta} \psi_{\alpha}^{a}(\mathbf{x}, t) \psi_{\beta}^{c}(\mathbf{x}, t) \psi_{\delta}^{b}(\mathbf{x}, t),
$$

where $\alpha, \beta$, and $\gamma$ are Dirac indices, $a, b$, and $c$ are colour indices, $\varepsilon$ is the Levi-Civita symbol, and $C$ is the charge conjugation operator. The projection of the correlation function on to vanishing spatial momentum is performed by the usual means of summing over $\mathbf{x}$ at $T=0$ where we imposed periodic boundary conditions in all directions. 
However, at finite temperature, anti-periodic boundary conditions must be imposed on quark fields in the Euclidean time direction. As a result, the screening correlator must be projected to the lowest Matsubara frequency [10]. The parity projection operators for the correlator whose propagation is measured in the direction $\mu$ is

$$
\mathcal{P}_{ \pm}^{\mu}=\frac{1}{2}\left(1 \pm \gamma^{\mu}\right)
$$

The group theory relevant for screening correlators is given in Appendix A

The zero momentum correlators of the two parities of the nucleon can be fitted to the behaviour expected of a Wilson fermion-

$$
\begin{aligned}
& S_{N_{+}}(t)=c_{+} \exp \left[-\bar{\mu}_{N_{+}} t\right]+c_{-} \exp \left[-\bar{\mu}_{N_{-}}\left(N_{t}-t\right)\right] \\
& S_{N_{-}}(t)=c_{-} \exp \left[-\bar{\mu}_{N_{-}} t\right]+c_{+} \exp \left[-\bar{\mu}_{N_{+}}\left(N_{t}-t\right)\right] .
\end{aligned}
$$

Because of the admixture from opposite parity states, these correlators change sign across the middle of the lattice. In this paper we used the absolute value of the correlators above. We note that $R_{N}$ does not require knowledge of $Z_{N}$ because the parity partners are generated by the same lattice operator. For the $T=0$ measurement, where $\bar{\mu}_{N^{ \pm}}=m_{N^{ \pm}}$. For the finite temperature measurement where we replace $t$ by $z$, we have

$$
\mu_{N^{ \pm}}^{2}=\bar{\mu}_{N^{ \pm}}^{2}-\sin ^{2}\left(\pi / N_{t}\right),
$$

when we use the prescription of [10].

\section{THE MESON SECTOR}

\begin{tabular}{|c|ccc|}
\hline$\kappa$ & $m_{\pi} / T_{c}$ & $m_{V} / T_{c}$ & $m_{A V} / T_{c}$ \\
\hline 0.1345 & $2.20 \pm 0.03$ & $3.55 \pm 0.06$ & $5.6 \pm 0.4$ \\
0.1347 & $1.99 \pm 0.03$ & $3.53 \pm 0.08$ & $5.5 \pm 0.5$ \\
\hline
\end{tabular}

TABLE III: Meson masses in units of $T_{c}$ at $T=0$ and $\beta=6.03$.

The analysis of meson masses at $T=0$ is absolutely standard. The results are collected in Table III We choose to express all results in units of $T_{c}$ for two reasons. First, because quenched QCD is not open to experimental tests and hence quoting numbers in $\mathrm{MeV}$ units is based on assumptions which cannot be tested. We prefer to quote ratios of quantities which are computable in practice. Second, because in quenched QCD the critical coupling is known with high precision, and $N_{t}=8$ is within the scaling region [28], the statistical and systematic errors involved in using this as a scale are completely under control.

\begin{tabular}{|c|ccc|}
\hline$m_{\pi} / T_{c}$ & $\mathrm{H}$ & $\mu_{H} / T_{c}$ & $\mu_{H} / m_{H}$ \\
\hline 2.20 & $\mathrm{PS}$ & $2.18 \pm 0.02$ & $0.99 \pm 0.02$ \\
& $\mathrm{~V}$ & $3.45 \pm 0.06$ & $0.97 \pm 0.02$ \\
& $\mathrm{AV}$ & $5.18 \pm 0.09$ & $0.93 \pm 0.06$ \\
\hline 1.99 & $\mathrm{PS}$ & $1.97 \pm 0.02$ & $0.99 \pm 0.02$ \\
& $\mathrm{~V}$ & $3.31 \pm 0.07$ & $0.94 \pm 0.03$ \\
& $\mathrm{AV}$ & $5.1 \pm 0.1$ & $0.92 \pm 0.08$ \\
\hline
\end{tabular}

TABLE IV: Meson screening masses, $\mu_{H}$ at $T=0.95 T_{c}$ in units of $T_{c}$ and the corresponding $T=0$ meson mass, $m_{H}$.

The analysis of most mesonic correlators below $T_{c}$ is equally straightforward. The only subtlety has been mentioned earlier: since the zero momentum screening correlator is measured for separations along the $z$-direction, the three polarizations states of the $\mathrm{V}$ and $\mathrm{AV}$ are two spatial and one temporal. The temporal polarizations have behaviour distinct from the spatial polarizations [9]. We measure the screening masses of the spatial polarizations only. Our main results for the screening masses below $T_{c}$ are summarized in Table IV] We discuss the relation between the screening mass and the pole mass in Appendix B Our results show that the pole mass of the mesons is hardly affected by temperature. 


\begin{tabular}{|c|cc|cc|}
\hline \multirow{2}{*}{$m_{\pi} / T_{c}$} & \multicolumn{2}{|c|}{$T=0$} & \multicolumn{2}{c|}{$T=0.95 T_{c}$} \\
\cline { 2 - 5 } & $E_{\text {int }} / m_{\pi}$ & $\chi^{2} /$ dof & $E_{\text {int }} / m_{\pi}$ & $\chi^{2} /$ dof \\
\hline 2.20 & $0.6 \pm 0.2$ & 1.92 & $-0.1 \pm 0.8$ & 0.25 \\
1.99 & $0.5 \pm 0.2$ & 1.28 & $0.1 \pm 0.7$ & 0.57 \\
\hline
\end{tabular}

TABLE V: $E_{\text {int }}$ in units of $m_{\pi}$ obtained by fitting the scalar correlator with the fit form in eq. (C1).

In Appendix [C we discussed the reasons why scalar decays are visible in quenched QCD, and how the final-state interaction energy, $E_{\text {int }}$, can be extracted. The analysis involves fitting the functional form in eq. (C1) at both $T=0$ and for $T<T_{c}$. The extracted physical parameters are given in Table $\mathbf{V}$. $E_{\text {int }}$ at $T=0$ is non-zero at the $95 \%$ significance level for both values of $m_{\pi}$ which we used. While the finite temperature measurements are statistically indistinguishable from these, they are consistent with zero within errors, due to the larger fit errors at $T>0$. It is interesting to note that the central values of $E_{\text {int }}$ at $T>0$ lie outside the $2 \sigma$ errors of the $T=0$ measurement.

The $\mathrm{SU}_{L}(2) \times \mathrm{SU}_{R}(2)$ chiral symmetry of the $\mathrm{QCD}$ vacuum is restored above $T_{c}$. The anomalous $\mathrm{U}_{A}(1)$ remains broken asymptotically, although its effects are seen to be small at temperatures of $1.5 T_{c}$. The most straightforward consequence of this is that the S/PS and the V/AV correlators should become pairwise degenerate in the high temperature phase. That this is the case is shown in Figure 1. The figure also shows that the correlation functions are not too far from those expected in a theory of non-interacting quarks (also called the free field theory, FFT).

At $T=0$ in the $\mathrm{V} / \mathrm{AV}$ sector we found $R_{V}=0.73 \pm 0.02$; at $T=0.95 T_{c}$ this drops marginally to $R_{V}=0.59 \pm 0.02$ for both the bare quark masses we have used. This indicates that chiral symmetry remains strongly broken up to $0.95 T_{c}$. A simple model of $\mathrm{V} / \mathrm{AV}$ mixing below $T_{c}$ was presented in [18] using a mixing parameter $\epsilon$, which can be adapted to our use by writing schematically

$$
S_{V}(T)=(1-\epsilon) S_{V}(0)+\epsilon S_{A V}(0), \quad \text { and } \quad S_{A V}(T)=(1-\epsilon) S_{A V}(0)+\epsilon S_{V}(0) .
$$

This gives $R_{V}(T)=(1-2 \epsilon) R_{V}(0)$. Using the values quoted above, we find $\epsilon=0.10 \pm 0.02$. In the unquenched theory one may expect much larger values of this parameter below $T_{c}$ [18]. We observed that at $T=1.5 T_{c}$ the value of $R_{V}$ drops as a power of the quark mass, vanishing as $m_{\pi}$ vanishes (see Figure 2). However, there is no dependence of $R_{S}$ on the quark mass.

We have extracted screening masses, $\mu_{H}$ from the correlation functions. We found that effective masses showed a good plateau which agreed with fits, unlike previous experience with Wilson quarks at $T>T_{c}$ [14. The reasons for this are explored in Appendix $B$ where some effective mass plateaus are also displayed. Our results for the screening masses are collected in Table VII The pairwise degeneracy of the masses is quite evident. It is also evident that the screening masses are close to that expected in a theory of free quarks. The screening masses in the S/PS channel are $7-9 \%$ smaller than that in the free theory, whereas in the V/AV channel they are within $2-3 \%$ of the free theory.

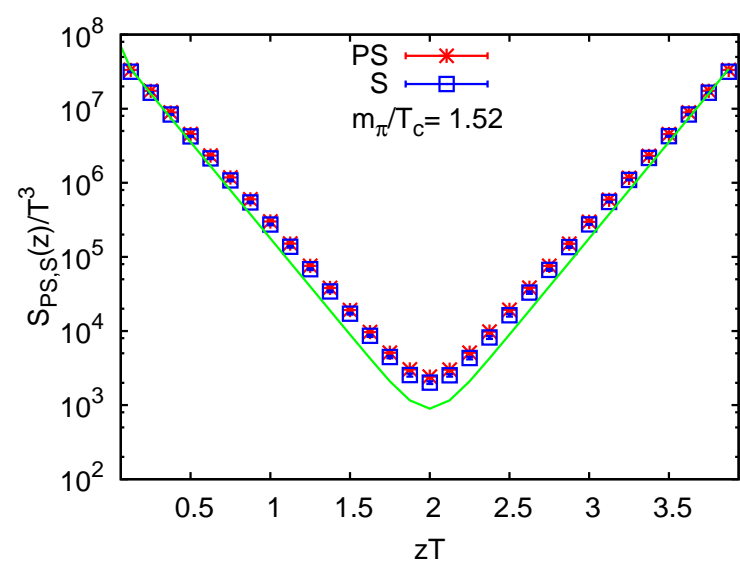

(a)

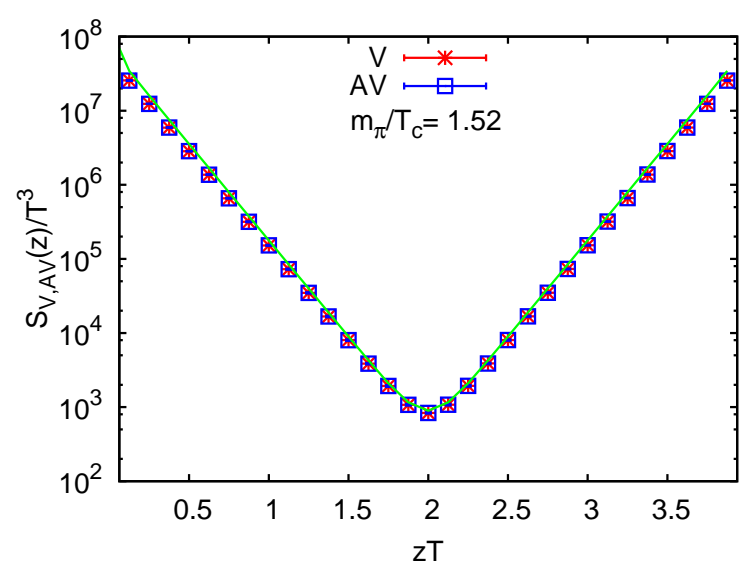

(b)

FIG. 1: A signal of restored chiral symmetry above $T_{c}$ is that (a) the PS and S correlators and (b) the V and AV correlators become degenerate. The continuous curves are correlation functions in a FFT; in order to remove trivial artifacts, these have been computed on a lattice of the same size. 


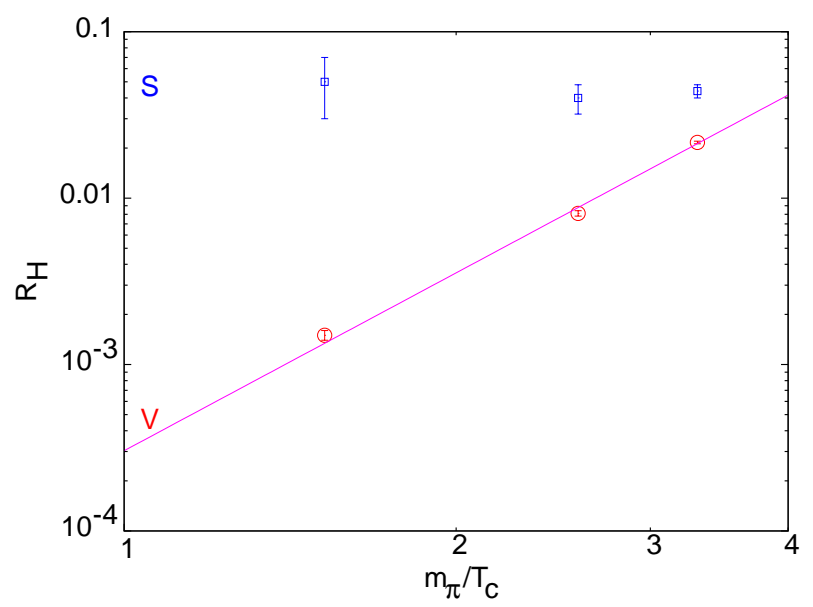

FIG. 2: Above $T_{c} R_{S}$ is found to be almost independent of $m_{\pi} / T_{c}$. However, $R_{V}$ goes to zero as a power of $m_{\pi} / T_{c}$, showing that correlation functions of the parity partners $V$ and $A V$ do become exactly degenerate in the limit.

\section{THE BARYON SECTOR}

Analysis of baryon correlators requires more care than meson correlators. This is because states of opposite parity contribute to each parity projected correlator, as shown in Figure 3. As a result, both the fitting procedure and the extraction of effective masses is more complex than the analysis for mesons. In addition, the projection of the screening correlator on to zero momentum requires a twist to compensate for the thermal boundary condition. The subsequent extraction of a screening mass from the nucleon correlator also requires the subtraction of eq. (10), resulting in additional loss of precision.

In Figure 3 it would appear that the finite temperature correlators are more nearly symmetric than those at $T=0$. Whether or not there is an early onset of chiral symmetry restoration can be probed by constructing the measure $R_{N}$, defined by eq. (6). At $T=0$ we find $R_{N}=0.88$ when $m_{\pi} / T_{c}=2.20$ and 0.89 at the lower quark mass. At finite temperature these change to $R_{N}=0.8$ and 0.83 respectively. Contrary to the visual impression created by Figure 3 , once the covariances between the $N_{ \pm}$correlators are accounted for, the correlation functions themselves do not show any tendency towards early restoration of chiral symmetry. In fact if we adapt the model of eq. (11) to this case, we find a mixing parameter $\epsilon=0.04$ in the nucleon sector, which is even smaller than that found for the V/AV at the same temperature.

The results of fitting the masses is shown in Table VI. Here we indeed find some precursor effects of chiral symmetry restoration in the form of a thermal shift in the mass splitting between the baryon and its parity partner. We measured

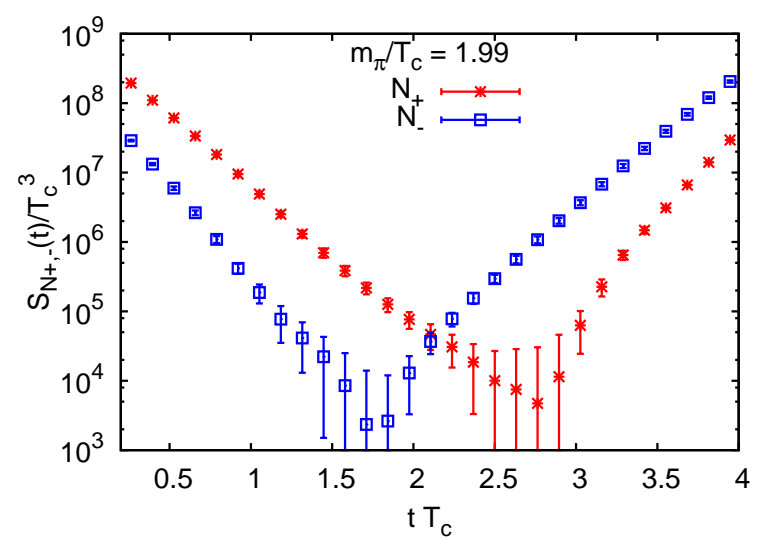

(a)

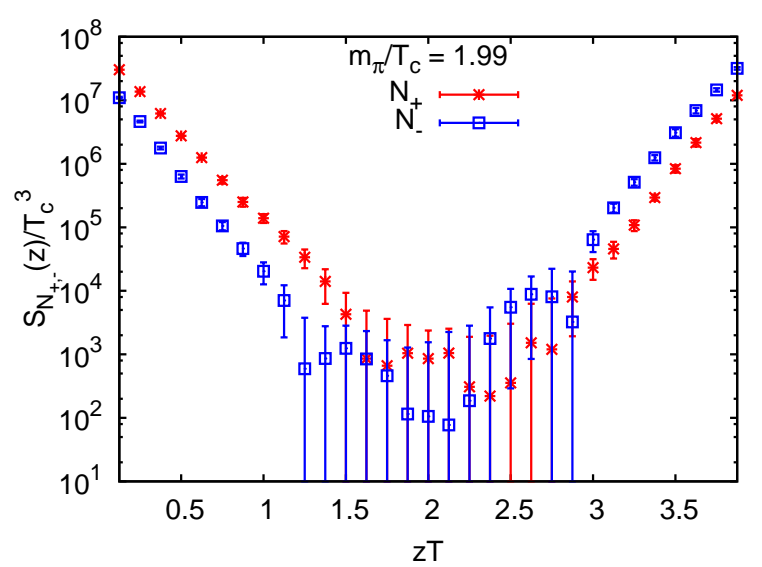

(b)

FIG. 3: Nucleon correlators at (a) $T=0$ and (b) $T=0.95 T_{c}$. Both sets are asymmetric, showing that the nucleon, $N_{+}$and its parity partner, $N_{-}$, are not degenerate. 


\begin{tabular}{|c|c|c|c|c|}
\hline$m_{\pi} / T_{c}$ & $m_{N_{+}} / T_{c}$ & $\mu_{N_{+}} / T_{c}$ & $m_{N_{-}} / T_{c}$ & $\mu_{N_{-}} / T_{c}$ \\
\hline 2.20 & $5.10 \pm 0.10$ & $5.3 \pm 0.2$ & $6.8 \pm 0.4$ & $6.6 \pm 0.4$ \\
1.99 & $4.9 \pm 0.1$ & $5.28 \pm 0.14$ & $6.7 \pm 0.5$ & $6.4 \pm 0.7$ \\
\hline
\end{tabular}

TABLE VI: Nucleon masses at $T=0$ and screening masses at $T=0.95 T_{c}$.

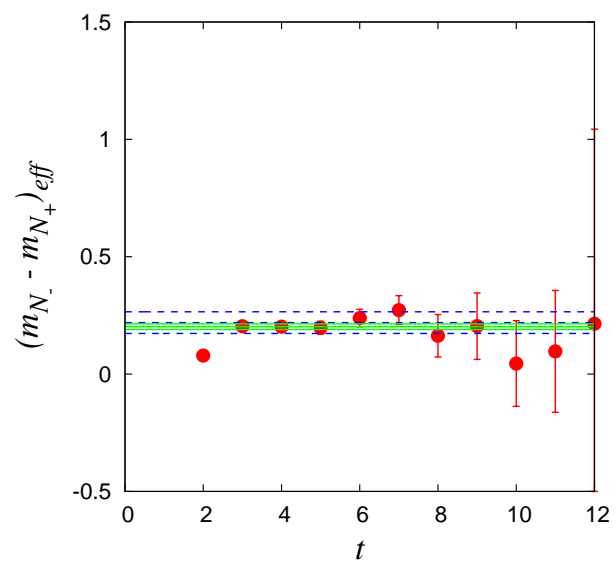

(a)

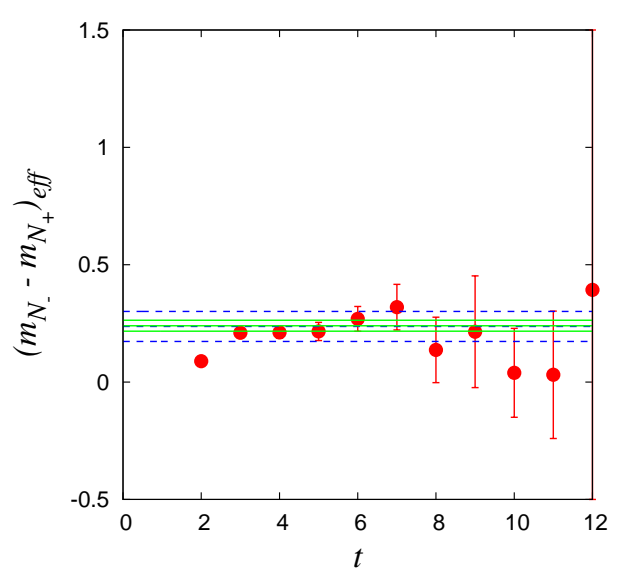

(c)

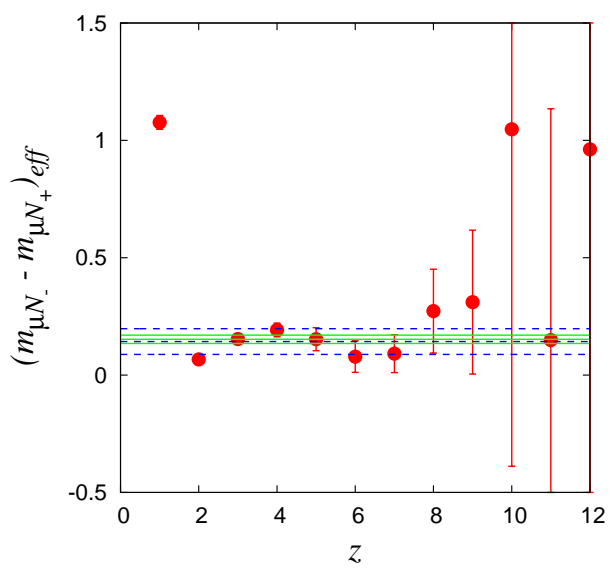

(b)

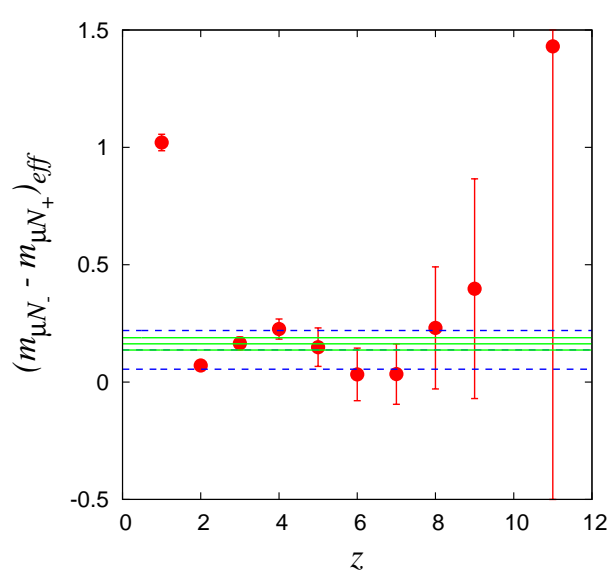

(d)

FIG. 4: The analysis of mass splittings between $N_{+}$and $N_{-}$for $m_{\pi} / T=2.20$ at (a) $T=0$ and (b) $T=0.95 T_{c}$ and for $m_{\pi} / T=1.99$ at (c) $T=0$ and (d) $T=0.95 T_{c}$. The data points are effective masses, the band between the dashed lines is obtained by taking the difference of screening masses. The band between the full lines is obtained by direct fits to the splitting between the two masses, and gives the results shown in eq. (12).

the splitting by the ratio of the correlators, $S_{N_{+}} / S_{N_{-}}$, and extract the mass difference, $\Delta m$, by fitting to an exponential form $\exp (-\Delta m t)$. Since this method takes care of covariances between the correlators $S_{N_{+}}$and $S_{N_{-}}$, we expect to control statistical errors better. The fit can be cross checked against the equivalent of effective masses for the ratio. Such checks are exhibited in Figure 4 . The resulting values are

$$
\frac{m_{N_{-}}-m_{N_{+}}}{T_{c}}=\left\{\begin{array}{ll}
1.53 \pm 0.09 & \left(m_{\pi} / T_{c}=2.20\right) \\
1.82 \pm 0.17 & \left(m_{\pi} / T_{c}=1.99\right)
\end{array} \quad \text { and } \quad \frac{\mu_{N_{-}}-\mu_{N_{+}}}{T_{c}}=\left\{\begin{array}{ll}
1.15 \pm 0.14 & \left(m_{\pi} / T_{c}=2.20\right) \\
1.24 \pm 0.20 & \left(m_{\pi} / T_{c}=1.99\right)
\end{array} .\right.\right.
$$

The thermal effect is significant, and leads to the results quoted in Table \. This is the most definite evidence to date about precursor effects to chiral symmetry restoration at $T_{c}$.

Above $T_{c}$ the correlators for $N_{ \pm}$become symmetric and degenerate. We find $R_{N}=0.230 \pm 0.005$ when $m_{\pi} / T_{c}=3.31$, $R_{N}=0.146 \pm 0.006$ for $m_{\pi} / T_{c}=2.58$ and $R_{N}=0.076 \pm 0.009$ for $m_{\pi} / T_{c}=1.52$. These lead to a vanishing of $R_{N}$ 
as a power of $m_{\pi} / T_{c}$. Chiral symmetry restoration is also seen in the fitted screening masses, displayed in Table VII. As in the meson sector, the screening masses are within 3-4\% of those expected in a theory of free quarks.

\section{SUMMARY AND CONCLUSIONS}

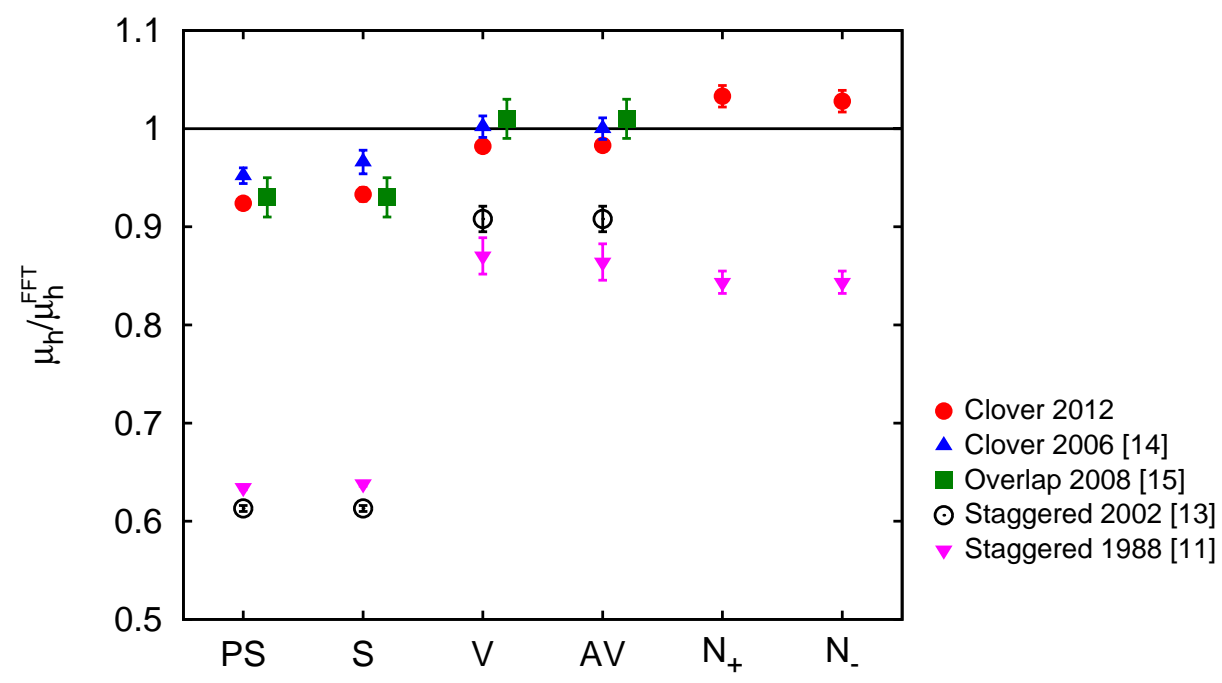

FIG. 5: The ratio of screening masses measured at $T=1.5 T_{c}$ in quenched QCD with those in FFT. Although all the results are not taken for exactly the same quark mass, the effects of the quark mass are very small (less than $1 \%$ in this study). The results of these present computations are in agreement, within $95 \%$ confidence limits, with those from earlier computations with clover [14] and overlap [15] quarks. However, the results from earlier computations with staggered quarks [11, 13] differ considerably, being further away from the limit of free fermions.

We have studied thermal correlations in quenched QCD in channels with various hadronic quantum numbers. The correlation functions are consistent with the usual picture of the QCD phase diagram- below $T_{c}$ long-distance correlations are mediated by hadrons and above $T_{c}$ these are mediated by weakly interacting quarks. It is approximately true that there are no thermal effects below $T_{c}$ (see Table I) and that above $T_{c}$ the effects seen are consistent with almost non-interacting quarks (see TableVII). However, there are several details which may impact our understanding of the thermal behaviour of QCD.

Above $T_{c}$, at the temperature $T=1.5 T_{c}$, we found strong signals of approximate chiral symmetry restoration in the near degeneracy of screening masses of hadronic parity partners, evident in Table VII. In addition we found, as before [12 16], that the correlation functions and screening masses are not far from those obtained in FFT, which is a model of non-interacting quarks.

Since there is persistent confusion in the literature regarding the S/PS channels, we made a survey of the literature and found systematic effects which we summarize in Figure 5 . This work uses the smallest lattice spacing used in a quenched study of screening to date, and agrees with previous work using clover quarks [14]. It also agrees with results of studies using overlap quarks at smaller lattice spacing [15]. However, it seems that studies with naïve staggered quarks in the quenched approximation always indicate stronger deviations from FFT than any of these above studies.

\begin{tabular}{|c|cccccc|}
\hline$m_{\pi} / T_{c}$ & $\mu_{P S} / T$ & $\mu_{S} / T$ & $\mu_{V} / T$ & $\mu_{A V} / T$ & $\mu_{N_{+}} / T$ & $\mu_{N_{-}} / T$ \\
\hline 1.52 & $5.54 \pm 0.02$ & $5.59 \pm 0.04$ & $5.88 \pm 0.02$ & $5.89 \pm 0.02$ & $8.72 \pm 0.10$ & $8.68 \pm 0.10$ \\
2.58 & $5.56 \pm 0.02$ & $5.59 \pm 0.02$ & $5.90 \pm 0.02$ & $5.91 \pm 0.02$ & $8.75 \pm 0.10$ & $8.73 \pm 0.10$ \\
3.31 & $5.61 \pm 0.02$ & $5.65 \pm 0.02$ & $5.95 \pm 0.02$ & $5.96 \pm 0.02$ & $8.82 \pm 0.08$ & $8.77 \pm 0.09$ \\
\hline
\end{tabular}

TABLE VII: Hadron screening masses at $T=1.5 T_{c}$ for three different masses of quarks. In FFT all the mesonic (baryonic) screening masses are expected to be 5.99 (8.44), 5.995 (8.456) and 6.01 (8.48) on our lattices, when the bare quark mass is tuned to give $m_{\pi} / T_{c}=1.52,2.58$ and 3.31 respectively. 
Since studies of screening with dynamical staggered quarks also claim such effects [12, 16], it is worth examining cutoff effects with staggered quarks more accurately in this context.

Even if the screening masses are close to that in the free theory at $T=1.5 T_{c}$, there are indications that there remain interesting physics in correlations. The deviation from FFT is the opposite of that seen in weak-coupling theory [29]. Using the measure $R_{S}, R_{V}$ and $R_{N}$ of eq. (6), we examined correlations in parity partner channels as a function of the quark mass (parametrized through the $T=0$ value of $m_{\pi}$ ). We found that as the quark mass is decreased $R_{V}$ and $R_{N}$ seem to vanish, as expected above $T_{c}$. However, $R_{S}$ does not depend on the quark mass, and remains non-zero. This still does not resolve the question of the relative importance of the multiple possible origins of this effect [16], that will depend on future temperature scans.

As highlighted in Table I mesons and the nucleon ground state are indeed unaffected by thermal effects below $T_{c}$. We also find that the splitting of the vector and axial vector meson masses is unaffected by raising the temperature to $T=0.95 T_{c}$. However, the opposite parity excitation of the nucleon is seen to move closer to the ground state. A closing of the mass gap between these two would signal chiral symmetry restoration, and that is expected to occur only at $T_{c}$. This is the first observation of precursors of the phase transition in the closing of the mass gaps of parity related states below $T_{c}$. No such effects have been seen earlier either in the glue sector or with quarks, nor do we see any effect in the V/AV channels here. Since the parameters $\epsilon$ introduced in eq. (11) is much smaller in the quenched theory than expected in QCD with dynamical quarks, perhaps one can expect the change in the mass gap to be more significant in the full theory.

A decreased splitting between the nucleon and its resonances implies that in a hadronic medium the interactions mediated through pions may keep the resonances in chemical equilibrium even after other baryons freeze out. This could result not only in changes in the net yield of nucleons, as seen at the LHC, but also possibly in the momentum distribution of nucleons. These implications clearly call for follow up lattice studies of nucleons below $T_{c}$.

Acknowledgements: The computations reported here were performed on the computing facilities of the ILGTI and the Department of Theoretical Physics, TIFR. We would like to thank Ajay Salve and Kapil Ghadiali for technical support. PM would like to acknowledge the Council of Scientific and Industrial Research (CSIR) for financial support through the Shyama Prasad Mukherjee fellowship. JM would like to acknowledge the hospitality of the Department of Theoretical Physics, TIFR, where he was a visiting fellow when he started working on this paper.

\section{Appendix A: Splitting of Wilson-Dirac fermions at finite temperature}

The point group of a $z$-slice of the lattice at finite temperature is the group $D_{4}$, which has 8 elements in 5 conjugacy classes. These classes are represented by the operators: the identity, $\pi / 2$ rotations about the $t$-axis, $R_{x y}, \pi$ rotations about the $t$-axis, $R_{x y}^{2}, \pi$ rotations about the $y$-axis, $R_{x t}^{2}$, and $\pi$ rotations about the $x+y$-axis, $R_{x+y}^{2}=R_{x y} R_{x t}^{2}$. Since the finite temperature theory is realized by periodic boundary conditions in the Euclidean time direction for bosons, the symmetry group for pure gauge operators is $D_{4}^{h}=D_{4} \times Z_{2}(T)$ where $T$ is the reflection operator in the $x y$ plane [8]. Since fermions are realized at finite temperature by anti-periodic boundary conditions, the $Z_{2}$ factor is not part of the symmetry group [9].

Acting on Dirac spinors, one can see immediately that

$$
R_{x y}=e^{i \pi \sigma_{12} / 4}=\frac{1}{\sqrt{2}}\left(1+i \sigma_{12}\right), \quad R_{x y}^{2}=i \sigma_{12}, \quad R_{x t}^{2}=i \sigma_{14},
$$

where $\sigma_{\mu \nu}=\left[\gamma_{\mu}, \gamma_{\nu}\right] / 2$ and $\gamma_{\mu}$ are the Euclidean Dirac gamma matrices. In the Euclidean chiral representation of the Dirac matrices, these three operators are block diagonal. The identity is always block diagonal, and $R_{x+y}^{2}$ is also block

diagonal since both $R_{x y}$ and $R_{x t}^{2}$ are. As a result, under the action of $D_{4}$, the 4-component Dirac spinor decomposes into two independent two-component objects, each corresponding to a specific helicity.

The $T=0$ parity operator is $P=\left(1+\gamma_{4}\right) / 2$. Since this is (block) off-diagonal in the chiral basis, it mixes different helicity components. In the chiral symmetry restored phase, the two helicity components give degenerate correlation functions and one of the parity eigenstates vanishes.

\section{Appendix B: Screening Correlator and the spectral function}

Here we compile some relevant formulae for understanding the screening correlation function. We take the $z$ direction as the direction of propagation, and examine correlators $S(z)$ with $z$ large.

The screening correlator is easy to understand in terms of the transfer matrix of a $z$ slice, and is therefore perfect for understanding the symmetries of the finite temperature transfer matrix. Information about thermal change of 
properties of hadrons, on the other hand, is easier to obtain from the real-time retarded correlator [30]

$$
G^{R}(x ; T)=i \theta\left(x^{0}\right)\langle[H(x), H(0)]\rangle_{T},
$$

where \langle\rangle$_{T}$ denotes thermal averaging at temperature $T$. The imaginary part of $G^{R}(p, T)$, the Fourier-transformed retarded correlator, is the spectral function $\sigma(p, T)$. A stable mesonic state, like the pion, contributes a term

$$
\sigma_{H}(p) \simeq A_{H} \epsilon\left(p_{0}\right) \delta\left(p^{2}-m_{H}^{2}\right)
$$

Here $A_{H}$ depends on the details of the operator. In particular, in our case of mesonic operators, $A_{H}$ depends on the nature of the source, and will be different for the wall and point sources. On the other hand, $m_{H}$ is the property of the hadronic state.

At temperatures $T$ sufficiently high but still below the transition temperature, one looks for thermal modifications of hadrons like a modification of the mass, or a thermal width. Such a modification may lead to, e.g., a relativistic Breit-Wigner form for the spectral function,

$$
\sigma_{H}(p ; T) \simeq Z \epsilon\left(p_{0}\right)\left(\frac{2}{\pi}\right) \frac{\Gamma m_{H}}{\left(p^{2}-m_{H}^{2}\right)^{2}+\Gamma^{2} m_{H}^{2}},
$$

with both $\Gamma$ and $m$ possibly dependent on $T$. A similar form of the spectral function is introduced at zero temperature for a resonance. In that case this form is useful when the unitarity of the S-matrix is not an issue. When it is, then a more careful analysis is required where the decay products of the resonance are explicitly included. The treatment of the scalar meson in Appendix $\mathrm{C}$ is an example.

If we ignore such issues, then the screening correlator is connected to the spectral function, as usual, through the integral [31]

$$
S(z, T)=\int_{-\infty}^{\infty} \frac{d p_{z}}{2 \pi} e^{i p_{z} z} \int_{0}^{\infty} \frac{2 d p_{0}}{p_{0}} \sigma\left(p_{0}, \overrightarrow{0}_{\perp}, p_{z} ; T\right) .
$$

It is easy to see that a change in the spectral function from the stable form eq. (B2) to a Breit-Wigner form eq. (B3) causes the large- $z$ behavior of the screening correlation function to change to

$$
S(z, t)=\frac{1}{2}\left[\mathrm{e}^{m_{H}\left(N_{L} / 2-z\right)} \cos \left(\frac{\Gamma z}{4}\right)+\mathrm{e}^{-m_{H}\left(N_{L} / 2-z\right)} \cos \left(\frac{\Gamma\left(N_{L}-z\right)}{4}\right)\right],
$$

assuming $\Gamma \ll m_{H}$. One can see from this that the effect of a width as small as $\Gamma / m \lesssim 0.1$, will be difficult to detect when the error bars are as small as a few percent. However, $\Gamma / m \simeq 0.2$ should be visible, provided the spectral function of eq. (B3) can be used.

On the other hand, the problem with the assumed Breit-Wigner form becomes clear if one examines effective masses instead of correlation functions. Then one finds that the effective masses would increase with $z$, contrary to the behaviour seen for the PS, V and AV. The reason is that in the lattice field theory, the unitarity of the S-matrix is enforced through the formulation. As a result, one cannot, in general, use a Breit-Wigner approximation to the spectral function in thermal QCD.

Next we make a detailed comparison of the behavior of the screening correlator with the corresponding correlators in FFT. We used wall sources for FFT, and compared the effective masses, $m(z)$, obtained on $32^{2} \times 8 \times N_{z}$ lattices (with $N_{z}=80$ ), with those obtained in the interacting theory.

In Figure 6] we compare the PS and V screening masses for our lightest quarks (corresponding to $m_{\pi} / T=1.52$ at $1.5 T_{c}$ ) to screening masses in FFT with similar bare quark mass. In the free theory, the screening correlator for wall source in these two channels are equal. The results for the FFT were obtained on a lattice with $N_{z}=80$ using the estimator $\ln C(z) / C(z+1)$ for the screening masses. The plateaus in the effective masses shown is due to the use of a wall source. When a point source is used, neither the free theory nor the interacting theory shows a plateau [14]. These checks give us confidence that we are able to extract the asymptotic behavior of the screening correlator.

The effective masses for the negative parity nucleon are also shown in Figure 6. In the interacting theory, a reasonable plateau is obtained in the effective mass, $C(z) \sim \exp \left(\mu(z) \times\left(N_{z}-z\right)\right)$. The plateau is more pronounced in our data for heavier quarks. We see that the effective mass in the interacting theory is larger than that in the free theory.

Since we use $N_{s} / N_{t}=L T=4$ we also check finite volume effects in a theory of free quarks. In Figure 7 we show the screening masses for pseudoscalar meson and nucleon on lattices with $N_{t}=8$ and varying $N_{s}$. With wall sources we find essentially no finite volume effect in either channels already for $L T=4$. Since finite volume effects are larger in the free theory than in the interacting theory, therefore finite volume effects in our studies are expected to be small. In contrast, point sources give large finite volume effects [14]. 

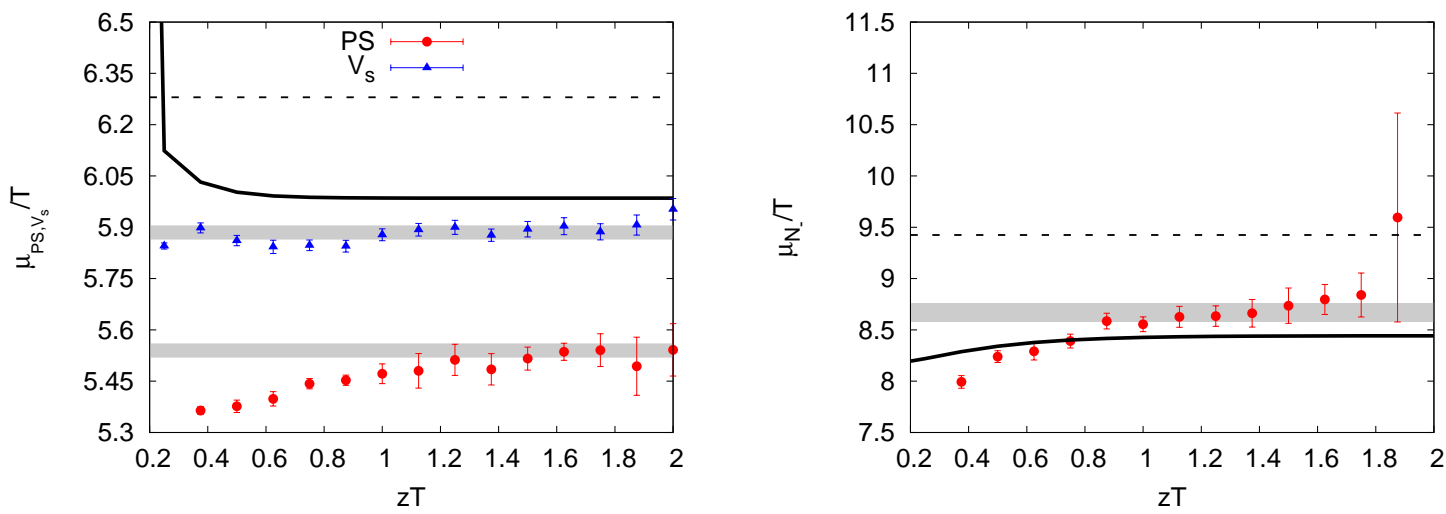

FIG. 6: Effective masses, in units of temperature, for meson and nucleon sources, at $T=1.5 T_{c}$ and in FFT. The black continuous curve denotes the FFT screening masses for $N_{t}=8$. The black dashed line shows the continuum value, $\mu=2 \pi T$ [12, 32]. The data points and the bands show the effective masses and the fitted estimate for the asymptotic value, respectively.
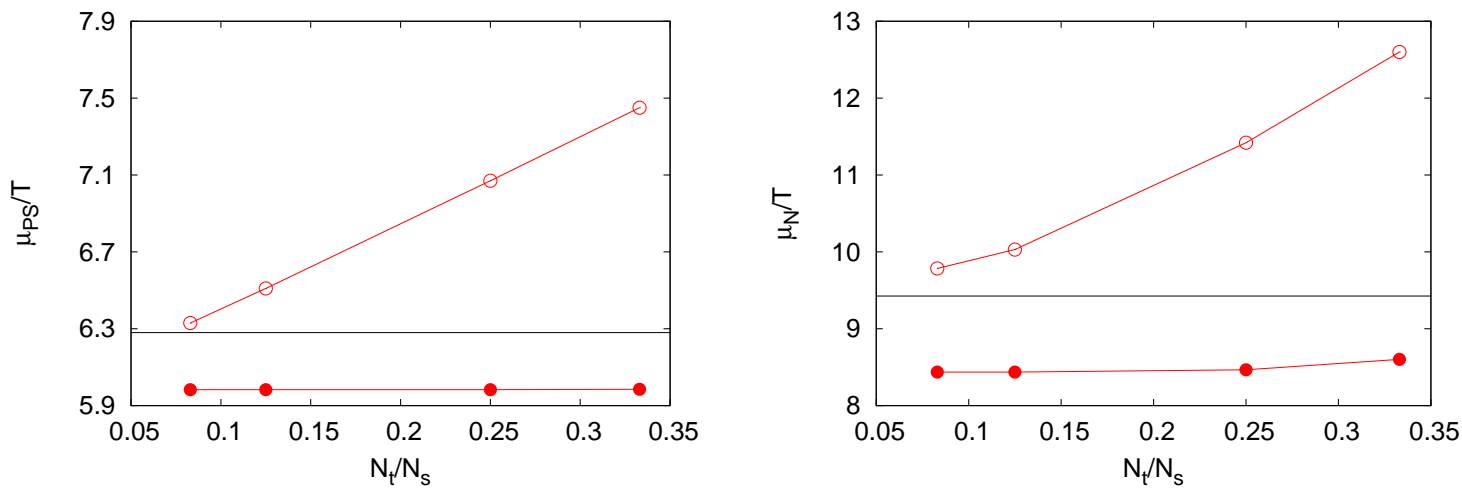

FIG. 7: Mesonic and nucleonic screening masses in FFT as a function of $N_{t} / N_{s}=1 / L T$ for $N_{t}=8$. The filled symbols are estimates from wall source quark propagators, while the unfilled symbols are estimated using point sources. In the latter case, since there is no plateau in the local mass, we use the prescription of [14] and use the local mass at distance $N_{z} / 4$.

\section{Appendix C: Scalar Meson}

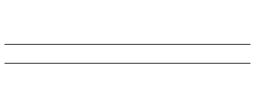

(a)

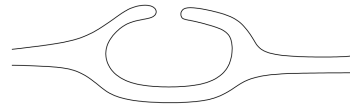

(b)

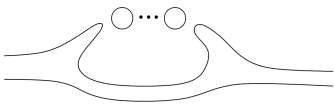

(c)

FIG. 8: Quark line diagrams which couples with scalar propagator in the full theory. In the quenched theory (c) is absent.

The non-trivial behaviour of quenched scalar correlators may be understood diagrammatically. In full QCD the scalar propagator has three kinds of topologies as shown in the figure above [33]. The third topology is absent in the quenched theory, as a result of which the scalar correlation function becomes negative when the quarks are light enough 33 35]. The missing diagram corresponds (for an isovector scalar) to the propagation of two different, but mass degenerate, pseudoscalars in the intermediate states; this is usually called a $\pi \eta^{\prime}$ (even in the two-flavour case). Due to the missing diagram, the quenched theory lacks reflection positivity.

In full QCD one would be able to fit the scalar propagator with a simple cosh term. However, in the quenched theory the contribution of the missing term has to be cancelled (a quenched ghost) [33]. This leads to a complicated 
spectral function resulting in the fit form which can be parametrized as 33 - 35

$$
\begin{aligned}
S^{Q \chi P T}(t)=b\{ & \left.\exp \left[-m_{S} t\right]+\exp \left[-m_{S}\left(N_{t}-t\right)\right]\right\} \\
& \quad-a_{S}\left\{\left(1+m_{\pi} t\right) \exp \left(-E_{\eta^{\prime} \pi} t\right)+\left(1+m_{\pi}\left(N_{t}-t\right)\right) \exp \left(-E_{\eta^{\prime} \pi}\left(N_{t}-t\right)\right)\right\} .
\end{aligned}
$$

The negative term is explicitly due to the quenched ghost. $m_{\pi / S}$ are the masses of the PS and S meson, $E_{\eta^{\prime} \pi}$ is the energy of the ghost state $\left(E_{\eta^{\prime} \pi}=2 m_{\pi}-E_{\text {int }}\right)$ and $-a_{S}$ is the coupling to the ghost. Since the ghost exactly cancels a physical term, these two parameters are physical. For sufficiently small $m_{\pi}$ the second term dominates at intermediate distances, leading to negative values for the correlator.

In our analysis of the scalar propagator we take the long-distance part of the propagator and extract from it both $a_{S}$ and $E_{\eta^{\prime} \pi}$. Using the renormalized propagator, we find the physical value of the coupling $a_{S}$. These results are reported in Table $\mathrm{V}$

The spectral function of the finite temperature scalar screening correlator is not known. However, as for the other states, we will assume that the form of the spectral function for $T<T_{c}$ is the same as at $T=0$, but the parameters could depend on $T$. With this assumption we will use the form in eq. (C1) at finite temperature below $T_{c}$.

[1] S. Gupta, X.-F. Luo, B. Mohanty, H.-G. Ritter and N. Xu, Science 332 (2011) 1525.

[2] J. Cleymans and K. Redlich, Phys. Rev. Lett. 81 (1998) 5248;

P. Braun-Munzinger and J. Wambach, Rev. Mod. Phys. 81 (2009) 1031.

[3] For a review see O. Philipsen, arXiv 1207.5999.

[4] H. Song, S. A. Bass, U. Heinz, T. Hirano and C. Shen, Phys. Rev. Lett. 106 (2011) 192301.

[5] A. Bzdak, V. Koch and V. Skokov, arXiv:1203.4529

[6] B. Grossman et al., Nucl. Phys. B 417 (1994) 289.

[7] P. B. Arnold and L. G. Yaffe, Phys. Rev. D 52 (1995) 7208.

[8] S. Datta and S. Gupta, Nucl. Phys. B 534 (1998) 392.

[9] S. Gupta, Phys. Rev. D 60 (1999) 094505.

[10] C. DeTar and J. B. Kogut, Phys. Rev. Lett. 59 (1987) 399, Phys. Rev. D 36 (1987) 2828.

[11] A. Gocksch, P. Rossi and Urs M. Heller, Phys. Lett. B 205 (1988) 334.

[12] K. D. Born et al.(MT ${ }_{c}$ Collaboration), Phys. Rev. Lett. 67 (1991) 302.

[13] R. Gavai, S. Gupta and P. Majumdar, Phys. Rev. D 65 (2002) 054506.

[14] S. Wissel et al., PoS LAT-2005, 164; S. Wissel, Ph. D dissertation (2006).

[15] R. Gavai, S. Gupta and R. Lacaze, Phys. Rev. D 78 (2008) 014502.

[16] M. Cheng et al., Eur. Phys. J. C 71 (2011) 1564;

D. Banerjee et al., Phys. Rev. D 83 (2011) 074510.

[17] M. Cheng et al., Nucl. Phys. A 862-863 (2011) 308.

[18] R. Rapp and J. Wambach, Adv. Nucl. Phys. 25 (2000) 1.

[19] M. Lüscher et al., Nucl. Phys. B 491 (1997) 323.

[20] B. Sheikholeslami and R. Wohlert, Nucl. Phys. B 259 (1985) 572.

[21] S. Gupta, Phys. Rev. D 64 (2001) 034507.

[22] G. Boyd et al., Nucl. Phys. B 469 (1996) 419.

[23] D. Becirevic et al., Phys. Lett. B 558 (2003) 69.

[24] M. Lüscher et al., Nucl. Phys. B 491 (1997) 344.

[25] G. P. Lepage and P. Mackenzie, Phys. Rev. D 48 (1993) 2250.

[26] M. Göckeler et al., Nucl. Phys. B (Proc. Suppl.) 53 (1997) 896.

[27] S. Sint and P. Weisz, Nucl. Phys. B (Proc. Suppl.) 63 (1998) 856.

[28] S. Datta and S. Gupta, Phys. Rev. D 80 (2009) 114504.

[29] M. Laine and M. Vepsäläinen, J. H. E. P. 02 (2004) 004.

[30] M. LeBellac, Thermal Field Theory, Cambridge University Press, Cambridge, England, (1996).

[31] F. Karsch and E. Laermann, in: Quark Gluon Plasma, vol. 3, edited by R. Hwa, World Scientific, Singapore.

[32] V. I. Eletskii and B. L. Ioffe, Sov. J. Nucl. Phys. 48 (1988) 384; W. Florkowski and B. L. Friman, Z. Phys. A 347 (1994) 271.

[33] W. Bardeen et al., Phys. Rev. D 65 (2001) 014509.

[34] N. Mathur et al., Phys. Rev. D 76 (2007) 114505.

[35] S. Prelovsek et al., Phys. Rev. D 70 (2004) 094503. 